\title{
A novel real-time monitoring system for a PVWPS
}

\author{
Amine Sallem ${ }^{1}$,Jean Marie Soubeiga ${ }^{2}$, Soufiane $\mathrm{Abi}^{3}$, Bachir Benhala ${ }^{3}$ and Nouri Masmoudi ${ }^{2}$ \\ ${ }^{1}$ ISGIS, University of Sfax, Tunisia \\ ${ }^{2}$ LETI-ENIS, University of Sfax, Tunisia \\ ${ }^{3}$ IESII, Faculty of Sciences, University of Moulay Ismail, Morocco
}

\begin{abstract}
The Tunisian Solar Plan, a renewable energy development plan elaborated by the government, foresees a $30 \%$ share of renewable in the electricity mix by 2030 . The solar photovoltaic capacity that can be installed for pumping water for irrigation is estimated at $24 \mathrm{MW}$ by 2030 . The water pumping system in the agricultural sector is considered among the main processes requiring the use of photovoltaic solar energy particularly in areas without an electric grid. The Photovoltaic Water Pumping System (PVWPS) contributes, in addition to the optimization of water pumping, to improve their energy performance in order to reduce their water consumption for irrigation. In Tunisia, PVWPS was adopted by the Ministry of Agriculture as a trustworthy alternative solution to cover the potable water needs of people in faraway areas, particularly in southern Tunisia. A novel real-time monitoring system for a PVWPS is proposed in this paper. The purpose of the monitoring system is to provide a useful tool for the operation and management of the PVWPS using the MODBUS communication protocol and the SIM900 module connected to ARDUINO to extend the control of the PV inverter to a distance greater than $1800 \mathrm{~m}$.
\end{abstract}

\section{Introduction}

Current energy consumption in agriculture is unsustainable. Modern agriculture production are highly dependent on fossil fuels, consuming about $30 \%$ of the world's available energy and producing more than $20 \%$ of world's greenhouse gas emissions [1]. In the next decades there will be significant and simultaneous increases in water, energy and food needs. These will lead to a degraded and depleted natural resource base, and increasing climate change challenges. Modernizing agriculture production by increasing the use of fossil fuels will be neither an affordable nor a sustainable option because of climate change and the impact of high and volatile fossil fuel prices on production costs and food prices.

To produce more food using less energy but also with cleaner sources of energy, food and agricultural systems will progressively need to decouple from fossil fuel dependence and adopt renewable energy sources [2]. The term "Renewable energies" includes all forms of energy: solar also known as photovoltaic energy, wind power, biomass, hydraulic [3]. These sources of energy are projected to assist in the improvement of the security of the worldwide energy supply system and in granting the developing nations with an opportunity to use affordable energy. For these reasons, the utilization of solar energy, known by photovoltaic systems, is rapidly turning into one of the most debated issues of the modern day. The production of electricity using photovoltaic systems is also an interesting possibility when farms are not connected to the electricity grid. Photovoltaic water pumping (PVWP) technology for irrigation is an innovative and sustainable solution to curb the grassland degradation. At the same time it can promote the conservation of farmland, especially in remote areas of Tunisia. In remote areas, the need of monitoring solar pumping control system is very necessary to ensure stable operation. This paper describes the hardware design for the solar pumping control system. The monitoring system is equipped with different sensors especially to measure the DC electrical output parameters of the PV panel and AC parameters of the inverter.

Previous work shows that PVWPS can be monitored using wireless sensor networks [4]. There are many drawbacks when it used to transmit data. In deed, it consumes more energy to operate.

Other transmission techniques can be used for monitoring the remote operated systems as Ethernet network (uses network cable to transmit data), RF module, Zigbee wireless network... But Ethernet network is affected by geographical environment [5], transmission bit error rate of RF module is high and less reliable [6] and Zigbee wireless network is more costly compared to other module [7].

GSM (Global System for Mobile Communication) is a standard set used to describe protocols for digital

\footnotetext{
*Corresponding author:sallem.amin@yahoo.com
} 
cellular networks. GSM network has low error rate, low costs and wide signal coverage. Users can communicate easily using GSM to perform monitoring anytime and anywhere.

A technical solution for the monitoring and tracking of PVWPS is shown in this work, and a novel quasi-realtime monitoring system for a PVWPS.

In this work, a stable and reliable system is built using ARDUINO microcontroller and GSM (SIM900) module to monitor and control performance of the solar pumping control system.

The figure below summarizes our objective for the proposed work:

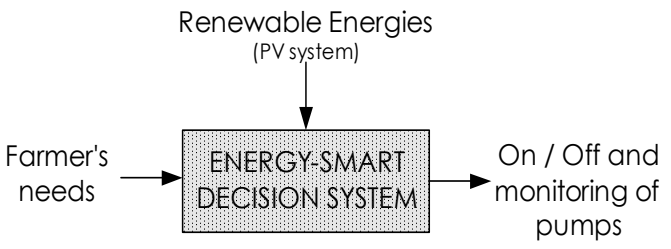

Fig. 1. Energy-smart decision system: The objective idea of the proposed work.

The rest of the paper is organized as follows: section 2 presents an overview of the Modbus communication protocol. Section 3 describes the experimental equipment used in this study. The development of the mobile application is presented in section 4. Finally, Section 5 presents the conclusions of this work and perspectives for future work.

\section{Modbus communication protocol}

The monitoring solar pumping control system prototype consists of smart mobile, ARDUINO MEGA, SIM900, GPS/GSM Neo7M module, MAX-485 TTL to RS-485 converter module, Solenoid valve connected to a relay card.

The user communicates with the microcontroller through SMS using the GSM network. A SIM card is installed in the GSM module which is on the field and connected to the microcontroller. The GSM module sends the data received to ARDUINO MEGA which also continuously receives the data from the relay card (Solenoid Valve are connected to a relay card to be controlled by ARDUINO) to check the condition of the motor pump.

The flow chart of the system is shown in fig. 2

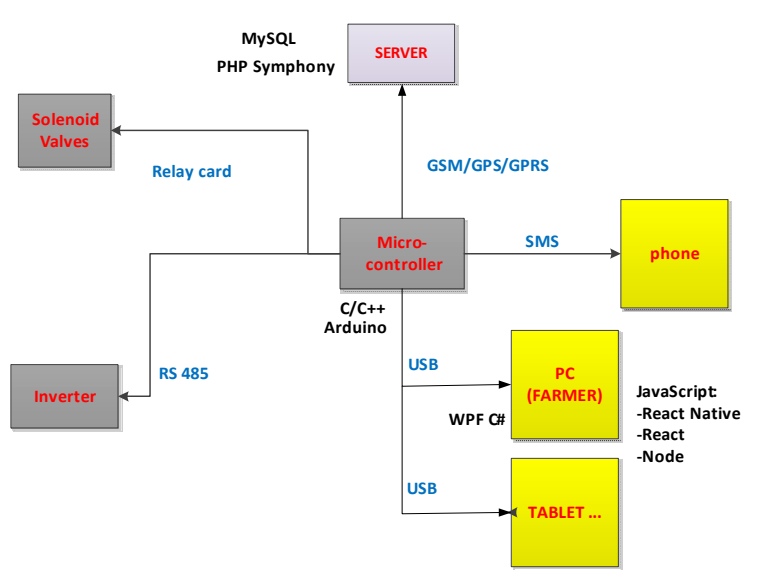

Fig.2. Monitoring solar pumping control system flow chart.

INVT inverter [8] was used in this work and it allows fairly direct control via the MODBUS communication protocol. Fig. 3, 4 and 5 show an example of communication and the type of control possible with this protocol.

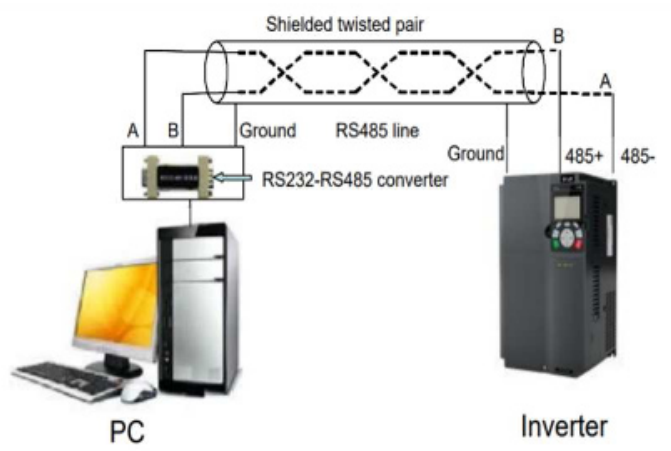

Fig. 3. Transmission technique using the MODBUS Protocol.

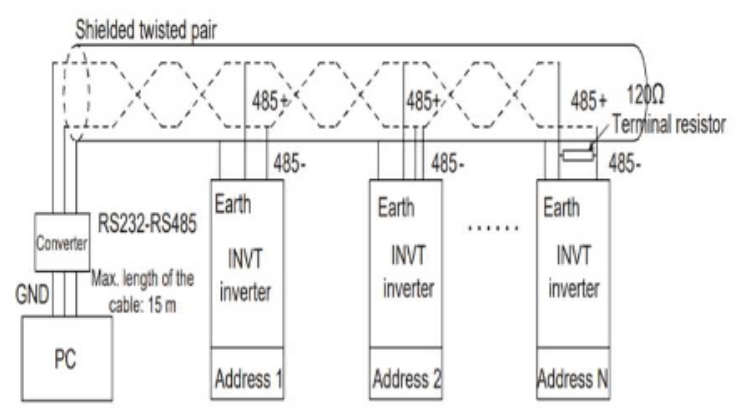

Fig. 4. A detailed example of MODBUS communication.

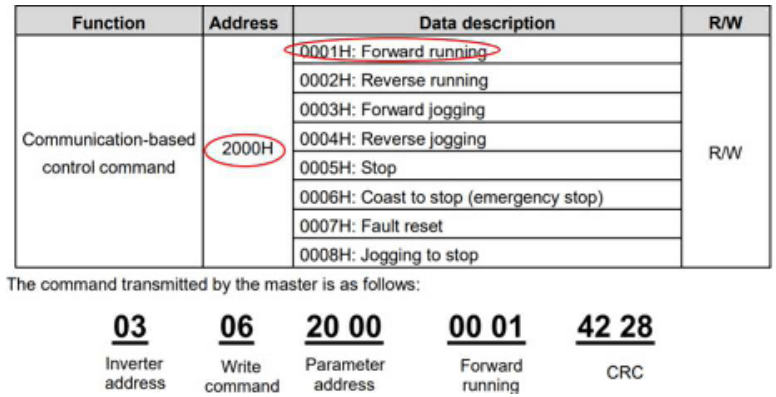

Fig. 5. Example of control command sent to start the inverter. 
In the next part, we will replace the computer (i.e., the master) by an ARDUINO MEGA where we will translate the command shown in Fig. 5.

\section{Experimental setup}

\subsection{ARDUINO board programming}

ARDUINO is an open-source platform and easy-to-use hardware and software. The selected ARDUINO MEGA is a microcontroller board based on the ATmega2560.

To facilitate sending commands to the INVT inverter through the ARDUINO MEGA, we will use the MODBUS MASTER library.

In the Fig. 6, we present the different module of the proposed monitoring solar pump control system.

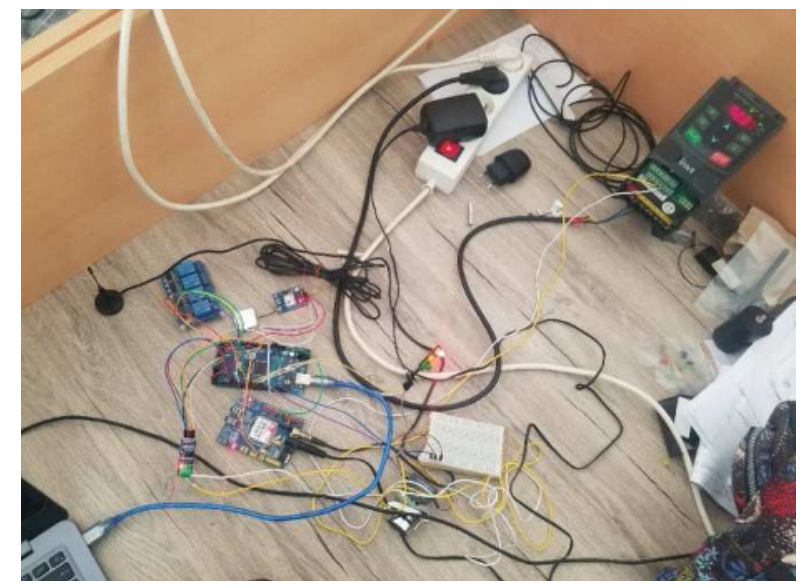

Fig. 6. Photo shows the proposed system, project end.

In addition, we can make the command by the computer and make a reading of the data.

The drive data on the ARDUINO board viewed through the computer are shown as follows:

-The operating frequency in $\mathrm{Hz}$ is: 20.50

-The set frequency in $\mathrm{Hz}$ is: 20.50

-The voltage in Volt is: 324.10

-Output voltage in Volt is: 129

-The output current in Ampere is: 0.00

-The speed of rotation in RPM is: 65535

-The output power is \%: 0.00

-The output torque is $\%: 6553.20$

\subsection{SIMCOM SIM900 Module}

SIMCOM SIM900 module is a GSM/GPS-compatible Quad-band cell phone, which works on a frequency of $850 / 900 / 1800 / 1900 \mathrm{MHz}$ [9]. We use this module, shown in Fig. 7, to receive and send text messages, from/to the user mobile phone.

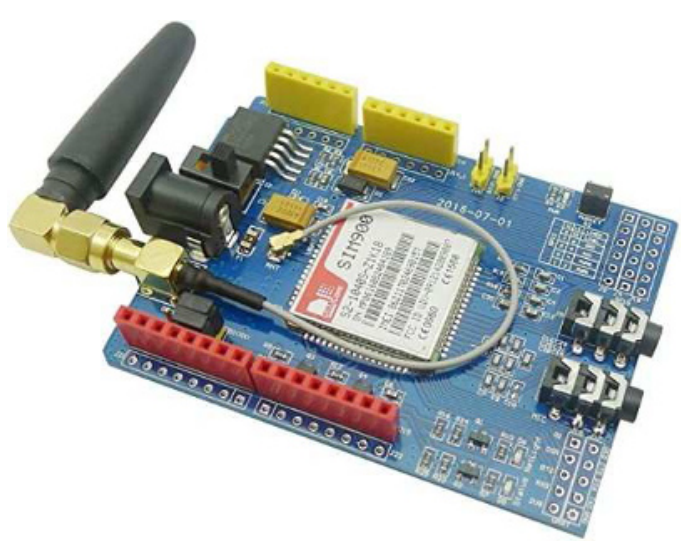

Fig. 7. SIMCOM SIM900 module.

Attention (AT) commands are used to control functionality of GSM modem. The different AT commands for module configuration are:

- ATD <number>: Checks number availability

- AT+CMGS: Send SMS

- AT+CSQ: Checking signal quality

- AT+CLTS: Get Local Timestamp

- AT+CREG: Network Registration Status

- AT+CCLK: Clock signal

- $\quad$ set the SIM900 to text mode: $\mathrm{AT}+\mathrm{CMGF}=1 \backslash \mathrm{r}$

- send SMS to a number: AT+CMGS = PHONE_NUMBER(in international format)

- read the first SMS from the inbox: $\mathrm{AT}+\mathrm{CMGR}=1 \backslash \mathrm{r}$

- read the second SMS from the inbox: $\mathrm{AT}+\mathrm{CMGR}=2 \backslash \mathrm{r}$

- read all SMS from the inbox: $\mathrm{AT}+\mathrm{CMGR}=\mathrm{ALL} \backslash \mathrm{r}$

\subsection{Relay module}

Relay module was connected to the ARDUINO MEGA and allows turning on and off the pump or any other devices, both AC and DC, based on the received SMS from the authorized user.

The orders received by the relay module that has been sent by ARDUINO are used to turn on or off the system controlling switch. Figure 8 shows photo for the prototype including the project and the user end. The smart mobile acts as user end.

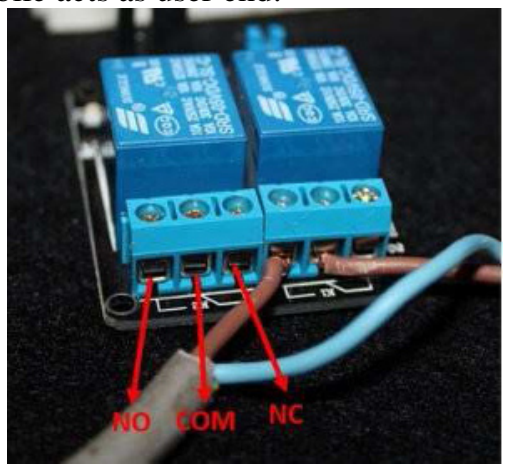

Fig.8. Relay Module 


\subsection{Ublox NEO-7M GPS Module}

Ublox NEO-7M is a pretty advanced GPS module recommend for user who are interested in navigation, tracking, the vast world of GPS. This module can receive data and then calculate the geographical position with a very high accuracy and speed. In addition the module has internal memory to save settings. This module is compatible with ARDUINO and can be used in any project. For this project, you will need parts which include:

- 1 Ublox Neo-7N GPS Module

- 1 Arduino MEGA

- Jumper Wires (Male to Female)

- 1 USB Data Cable

- 1 Female SMA antenna (optional; if needed) Utilising one main library, the TinyGPS++ library by Mikal Hart, it help massively in using this Ublox GPS module with ARDUINO.

The pinout of this module is presented in the figure below.

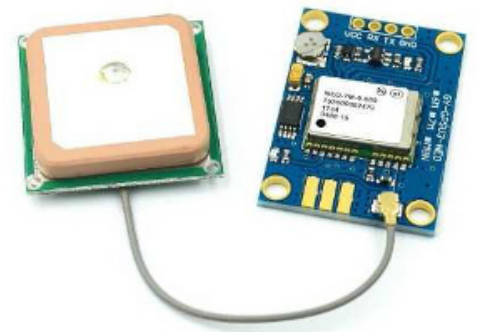

Fig.9. NEO-7M GPS Module

\section{Mobile application development}

In this section, a mobile application with REACT NATIVE [10] has been developed and it is compatible with IOS and ANDROID for controlling the variable speed drive.

We will use the direct sending of SMS through the phone without going through the writing phase, the user will just have to click on the different keys for the command. In our example we will take the case of a user with two watering sites.

The first pages (images in figure 10) are dedicated to configuring the application. The user (farmer) gives:

- The number of sites that the farmer owns

- The name of its sites

- The number of pumps he owns per site

- The name assigned to the pump as well as its module number.

Let us illustrate this configuration through a user who owns 02 sites: Sfax (in the south of Tunisia) and Tunis (in the north).

\# Sfax: 02 pumps

$\square$ Name of Pump1: Watering vegetables

Pump 1 number: 95788294

$\square$ Name of Pump 2: Watering the flowers

Pump 2 number: 95788294

\# Tunis: 01 pump

$\square$ Name of Pump 1: Fruit watering

Pump 1 number: 95788294.

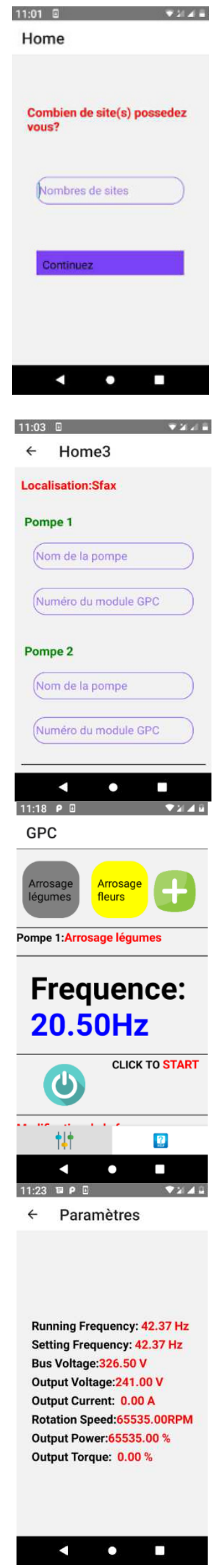

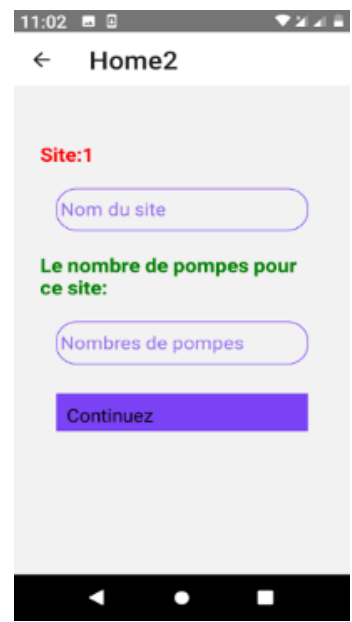
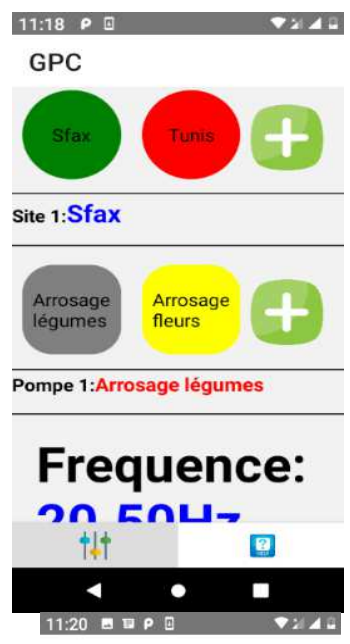

GPC

Modification de la frequence

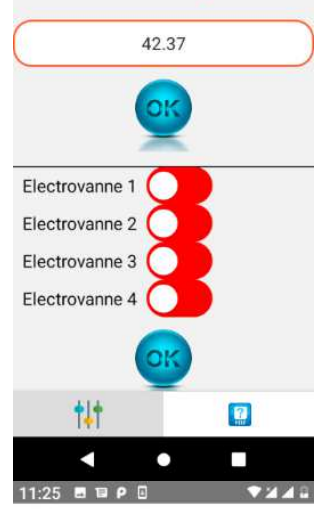

GPC
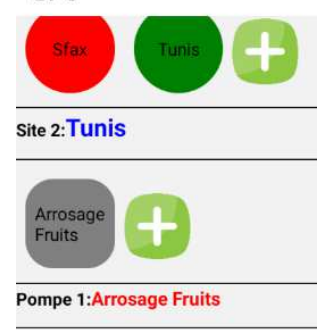

Frequence: 42.37Hz 
Fig. 10. Extract from the application developed in React Native.

The user can thus on the home page:

- Set the frequency of each pump

- Activate / Deactivate the solenoid valves

- Start / Stop the pump

- View the parameters of each pump (voltage, current, speed, frequency)

\section{Conclusion}

PVWPS offers an alternate means to meet the electricity demand for irrigation and livestock watering. Under the circumstances of inadequate supply of conventional sources of energy, the solar water pump can play a significant role.

In this present work, we have realized a novel real-time monitoring system based on Simcom SIM900 module and ARDUINO broad, and by a mobile application development for Photovoltaic Water Pumping System (PVWPS). We have monitored by sending and receiving SMS through the variable-speed drives of the Sfax and the Tunis watering sites.

In our future work, we will try to improve the general vision of the study by integrating the geolocation of reading, sending of data to the server and the development of a web site for the administrator and the clients.

\section{References}

1. W. Yamaka, R. Phadkantha, and P. Rakpho, "Economic and energy impacts on greenhouse gas emissions: A case study of China and the USA," Report Energy, 6th International Conference on Advances on Clean Energy Research, ICACER 2021 April 15-17, 2021, Barcelona, Spain

2. John Vourdoubas, Olivier Dubois, Energy and agrifood systems: production and consumption, Mediterra 2016, Chapter 7, pp. 159-179, Presses de Sciences Po, Paris, 2016.

3. R Sims, A Flammini, M Puri, S Bracco, "Opportunities for agri-food chains to become energy-smart", UN Food and Agricultural Organisation, 2015, Rome and GIZ. ISBN: 978-92.

4. Ranhotigamage C., and Mukhopadhyay, S. C., 2011. Field trials and performance monitoring of distributed solar panels using a low-cost wireless sensor network for domestic application. IEEE Sensors Journal 11, 2583-2590.

5. Dargie, W., and Poellabauer, C., 2010 Fundamentals of Wireless Networks: Theory and Practice, Wiley, NJ, USA.

6. Liu Hui, Wang Mao-hua, and et al., 2008. Development of farmland soil moisture and temperature monitoring system based on wireless sensor network. Journal of Jilin University (Engineering and Technology Edition). 604-608.

7. Junguo ZHAN, Wenbin LI, Ning HAN, Jiangming KAN, 2008. Forest fire detection system based on
Zigbee wireless sensor network. Journal of Beijing Forestry University. 122-124.

8. http://www.invt.su/assets/files/GD350/ruk_na_exp 1GD350.pdf

9. https://simcom.ee/documents/SIM900/SIM900_A T\%20Command\%20Manual_V1.11.pdf

10. https://reactnative.dev/docs/getting-started 